\title{
Echo spectroscopy and Atom Optics Billiards
}

\author{
M. F. Andersen, A. Kaplan, T. Grünzweig and N. Davidson. \\ Department of Physics of Complex Systems, Weizmann Institute of Science, \\ Rehovot 76100, Israel
}

\begin{abstract}
We discuss a recently demonstrated type of microwave spectroscopy of trapped ultra-cold atoms known as "echo spectroscopy" [M.F. Andersen et. al., Phys. Rev. Lett., in press (2002)]. Echo spectroscopy can serve as an extremely sensitive experimental tool for investigating quantum dynamics of trapped atoms even when a large number of states are thermally populated. We show numerical results for the stability of eigenstates of an atom-optics billiard of the Bunimovich type, and discuss its behavior under different types of perturbations. Finally, we propose to use special geometrical constructions to make a dephasing free dipole trap.
\end{abstract}

Key words:

PACS:

\section{Introduction}

The subject of decoherence of a quantum system has witnessed a renewed interest in the last years. In addition to being central to our understanding of the transition from quantum to classical physics[1], it is of outmost importance in the blooming field of Quantum Information[2], where decoherence or dephasing represent loss of information.

A quantum superposition state "collapses" due to the dissipative interaction with the environment (e.g. in the case of trapped atoms, the coupling to the electro-magnetic vacuum leads to spontaneous scattering of photons). This interaction is irreversible in nature. On the other hand, the macroscopic (ensemble averaged) response of a quantum system prepared in a superposition state decays due to the dephasing resulting from local variations in the evolution of the system (e.g. inhomogeneous broadening). By stimulating an effective "time reversal", revivals of coherence in dephased systems (at least partial ones) have been reported for spin echoes [3] and photon echoes $[4,5]$, 
and more recently for a motional wave packet echo using ultra cold atoms in a one-dimensional optical lattice [6].

In this paper we discuss the technique of "echo spectroscopy" on ultra cold atoms trapped in an optical dipole trap, which utilizes a micro wave (MW) analog to the photon echo [7]. We show that this technique can be used to study the quantum dynamics of trapped atoms, even when many states are thermally populated. We show that the stability of eigenstates under perturbations of the Hamiltonian plays an important role for the echo signal. Numerical calculations of this quantity for an atom-optics Bunimovich stadium are presented. Next, by overcoming the inhomogeneous shift, which is the main limiting factor for the coherence time in dipole traps [8], echo spectroscopy opens the way for using dipole trapped atoms in quantum information and possibly for high precision spectroscopy. Finally, our analysis leads to a proposal of a dephasing free dipole trap.

\section{Principles of Echo Spectroscopy}

One of the most widely used MW spectroscopy techniques is the so called Ramsey method [9]. It works on two level atoms after the following principle: The atoms are prepared in one of their two states (denoted $|1\rangle$ ). Next, a short MW-pulse close to resonance transfers the atoms into a coherent superposition state of the two levels (This pulse is usually called a $\frac{\pi}{2}$ pulse), which is left to evolve for a certain time, without MW-irradiation. Due to the detuning between the atomic resonance frequency and the MW-frequency, the time evolution will cause an atomic phase to develop in the rotating frame of the MW field. A second $\frac{\pi}{2}$-pulse maps this phase onto populations of the two levels, which can be detected. If the MW-frequency is scanned, this phase difference is scanned, and sinusoidal oscillations in the populations, known as Ramsey fringes, are seen. When Ramsey spectroscopy is performed on an ensemble of inhomogeneously broadened two level atoms, each atom will acquire a slightly different phase in the dark period, causing the macroscopic fringe contrast to decay when increasing the time between the $\frac{\pi}{2}$-pulses. Thus the Ramsey fringe contrast is a measure of the dehpasing of the system.

The dephasing can be reversed by stimulating a coherence echo. This is done by adding a population inverting pulse (a $\pi$-pulse) between the two $\frac{\pi}{2}$-pulses. If the $\pi$-pulse is added exactly in the middle between the two $\frac{\pi}{2}$-pulses each part of the superposition state generated by the first $\frac{\pi}{2}$-pulse will have spent an equal amount of time in both levels at the time of the second $\frac{\pi}{2}$-pulse, and will therefore be exactly in phase with each other. This means that a coherence echo have appeared at the time of the second $\frac{\pi}{2}$-pulse even for a system that have dephased completely before the $\pi$-pulse. When measuring 
the populations the coherence echo is therefore observed by seeing that all the atoms have returned to the initial state after the $\frac{\pi}{2}-\pi-\frac{\pi}{2}$-pulse sequence. If the time $\left(\tau_{1}\right)$ between the first $\frac{\pi}{2}$ and the $\pi$-pulse is kept constant, and the time between the $\pi$ and the second $\frac{\pi}{2}$-pulse $\left(\tau_{2}\right)$ is swept, the coherence echo is seen as a decrease in the population of $|2\rangle$ for $\tau_{2}=\tau_{1}$ (see Fig. 1). The population $P_{2}$ for $\tau_{2}=\tau_{1}$ is referred in what follows as the "echo signal", where $P_{2}=0$ indicates full revival of coherence and $P_{2}=\frac{1}{2}$ indicates complete dephasing.

Experimental data shown in this paper is taken using two hyperfine levels of the ground state of ${ }^{85} R b$ atoms trapped in a dipole trap. These two levels $\left(\left|5 S_{1 / 2}, F=2, m_{F}=0\right\rangle\right.$, denoted $|1\rangle$, and $\left|5 S_{1 / 2}, F=3, m_{F}=0\right\rangle$, denoted $\left.|2\rangle\right)$ are separated by the energy difference $E_{H F}=\hbar \omega_{H F}$ where $\omega_{H F}=2 \pi \times 3.036$ $\mathrm{GHz}$ is the hyperfine splitting. For spectroscopy of trapped atoms we must consider the entire Hamiltonian including both the internal and the external degrees of freedom of the atom. Since the dipole potential is inversely proportional to the trap laser detuning $\delta[10]$ there is a slightly different potential for atoms in different hyperfine states $\$ and the Hamiltonian of our trapped two level atom can be written as:

$$
\begin{aligned}
H & =H_{1}|1\rangle\left\langle 1\left|+H_{2}\right| 2\right\rangle\langle 2| \\
& =\left(\frac{p^{2}}{2 m}+V_{1}(\mathbf{x})\right)|1\rangle\left\langle 1\left|+\left(\frac{p^{2}}{2 m}+V_{2}(\mathbf{x})+E_{H F}\right)\right| 2\right\rangle\langle 2|,
\end{aligned}
$$

where $p$ is the atomic center of mass momentum and $V_{1}\left[V_{2}\right]$ the external potential for an atom in state $|1\rangle[|2\rangle]$, much smaller than $E_{H F}$. The atoms are initially prepared in their internal ground state $|1\rangle$. Their total wave function can be written as $\Psi=|1\rangle \otimes \psi$, where $\psi$ represents the motional (external degree of freedom) part of their wave function. If a MW field close to resonance with $\omega_{H F}$ is applied, transitions between the eigenstates of the Hamiltonian corresponding to different internal states, can be driven. The small size of optical traps (typically on the order of $100 \mu \mathrm{m}$ or less) as compared to the MW wavelength $(\sim 10 \mathrm{~cm})$, ensures that the momentum of the MW photon can be neglected (Lamb-Dicke regime [10]). Even though the internal states of the atoms are described as a two-level system Eq.1 describes a multi-level system in two energy manifolds. When the MW-field is added to the Hamiltonian in Eq.1 and the time-dependent Schrödinger equation is formulated it is seen that the transition matrix elements between eigenstates of Eq. 1 are given by $C_{n n^{\prime}}=\left\langle n^{\prime} \mid n\right\rangle \times M_{1 \rightarrow 2}$ where $M_{1 \rightarrow 2}$ is the free space matrix element for the internal state transition, and $\left\langle n^{\prime} \mid n\right\rangle$ is the overlap between the initial motional eigenstate of $H_{1}$ and an eigenstate of $H_{2}$. Therefore, when a strong and short MW pulse is applied, the motional part of the

$\overline{1}$ For these two hyperfine levels the matrix elements for the dipole interaction are identical, hence the relative difference between the two optical potentials is $\left(V_{1}-V_{2}\right) / V_{2} \simeq \frac{\omega_{H F}}{\delta} \sim 10^{-3}$ for our experimental parameters. 


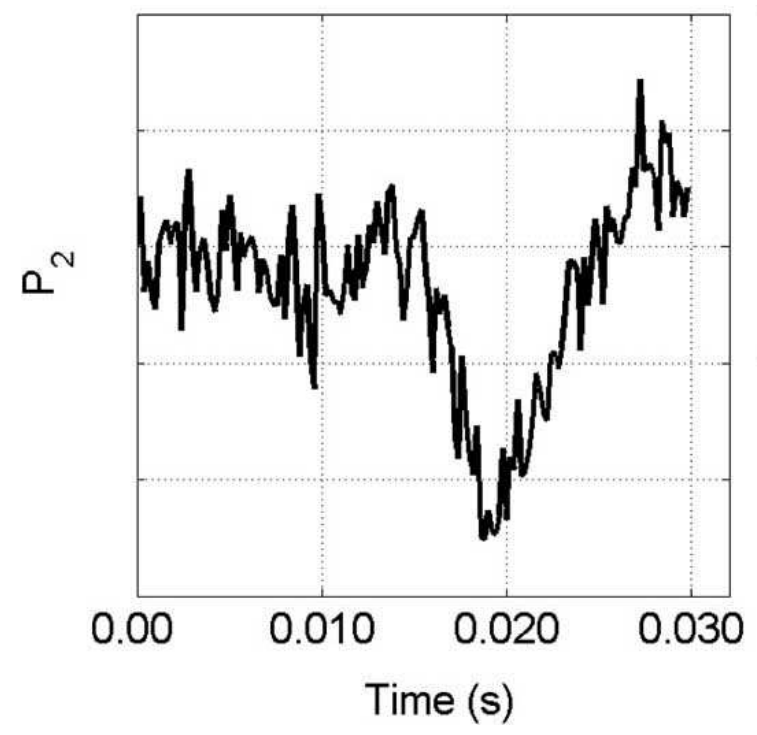

Fig. 1. Echo signal $\left(P_{2}\right)$ measured as a function of time between $\pi$-pulse and second $\pi / 2$-pulse $\left(\tau_{2}\right)$ for fixed $\tau_{1}$. A dip in $P_{2}$ is seen, showing a "coherence echo". $\tau_{2}=\tau_{1}$

initial wave function is simply projected into $V_{2}(\mathbf{x})$. If $V_{1}(\mathbf{x})=V_{2}(\mathbf{x})$ then clearly $\left\langle n^{\prime} \mid n\right\rangle=\delta_{n n^{\prime}}$. For a small enough potential difference (perturbation) $\delta V=V_{2}(\mathbf{x})-V_{1}(\mathbf{x})$ the approximation $\left\langle n \mid n^{\prime}\right\rangle \simeq \delta_{n n^{\prime}}$ is valid. In the general case, a projected eigenstate of $H_{1}$ will not be an eigenstate of $H_{2}$, and therefore will evolve in the new potential, causing the overlap $|\langle n(t=0) \mid n(t=\tau)\rangle|$ to decay $\left(|n(t=\tau)\rangle \equiv \exp \left(-i \frac{H_{2}}{\hbar} \tau\right)|n\rangle\right)$.

If Ramsey spectroscopy is performed on atoms initially prepared in an eigenstate of $V_{1}$ characterized by the quantum number $n$, then the probability to be in the internal state $|2\rangle$ after the $\pi / 2-\pi / 2$ pulse sequence can be shown to be:

$$
P_{2}=\frac{1}{2}\left(1+\left\langle n\left|e^{-i\left(\frac{H_{2}}{\hbar}-\omega_{M W}+\Delta_{n}\right) \tau}\right| n\right\rangle \cos \left(\Delta_{n} \tau\right)\right)
$$

where $\omega_{M W}$ is the MW-frequency, $\tau$ is the time between the pulses, and $\Delta_{n}$ is a generalized, state-dependent, detuning (it reduces to the detuning when only two levels are coupled by the MW-field), defined so $\left\langle n\left|\exp \left(-i\left(\frac{H_{2}}{\hbar}-\omega_{M W}+\Delta_{n}\right) \tau\right)\right| n\right\rangle$ is real and positive. Scanning $\omega_{M W}$ for a fixed $\tau$ yields the usual Ramsey fringes with a contrast given by $\left\langle n\left|\exp \left(-i\left(\frac{H_{2}}{\hbar}-\omega_{M W}+\Delta_{n}\right) \tau\right)\right| n\right\rangle=|\langle n(t=0) \mid n(t=\tau)\rangle|$. Since $\left\langle n\left|\exp \left(-i\left(\frac{H_{2}}{\hbar}-\omega_{M W}+\Delta_{n}\right) \tau\right)\right| n\right\rangle=\left|\left\langle n\left|\exp \left(i\left(\frac{H_{1}}{\hbar}\right) \tau\right) \exp \left(-i\left(\frac{H_{2}}{\hbar}\right) \tau\right)\right| n\right\rangle\right|$ this contrast serves as a measure of the stability of the quantum evolution under a small perturbation in the Hamiltonian [11].

In our experiments atoms are not initially in a single motional eigenstate, but a thermal ensemble of atoms incoherently populates many eigenstates. The total population in $|2\rangle$ is then given by an average of $P_{2}$ over the initial thermal 
ensemble. $\Delta_{n}$ depends on the initial state, and due to this spread in detuning the fringe contrast of the ensemble-averaged $P_{2}$ decays rapidly even when $|\langle n(t=0) \mid n(t=\tau)\rangle| \simeq 1$ for all populated states (note the analogy to the inhomogeneous broadening of two level atoms). Hence, the decay of the fringe contrast in Ramsey spectroscopy of thermal trapped atoms does not teach us anything about the dynamics in the trap $(|\langle n(t=0) \mid n(t=\tau)\rangle|)$ but simply measures the spread in $\Delta_{n}$. This spread also limits the interrogation time for high precision spectroscopy.

Equation 1 indicates that if a special trap can be constructed so that $\Delta_{n}$ is independent of $n$, then the fast decay of the Ramsey fringes will be suppressed. An example of such a dephasing-free trap is an inverted pyramid of exponentially decaying evanescent waves, in which gravity provides the vertical confinement. This trap have the special feature that $V_{2}(\mathbf{x})$ is exactly equal to $V_{1}(\mathbf{x})$ shifted along the vertical axis. Therefore all eigenenergies are shifted the same amount and $\Delta_{n}$ is indeed a fixed number. This makes such a trap an excellent candidate for high precision spectroscopy. Note, that in order to use traps for this kind of measurements, the suppression of the relative spread in $\Delta_{n}$, should be accompanied by a reduction in their average value, e.g. by increasing the trap's detuning. Since the proposed trap is a "dark optical trap", where atoms are trapped by repulsive light forces mainly in the dark [12], hence minimizing the amount of interaction with the trap's light, then in addition to being dephasing-free it has also a low photon scattering rate (which will now be the limiting factor for the coherence time) and a small $\Delta_{n}$.

Apart from using special geometries a possible solution to the above mentioned dephasing is to reverse it using "echo spectroscopy" by adding a MW $\pi$-pulse between the two $\frac{\pi}{2}$ pulses. $P_{2}$ for atoms initially populating a single eigenstate then becomes:

$$
\begin{aligned}
P_{2} & =\frac{1}{2}\left[1-\operatorname{Re}\left(\left\langle n\left|e^{i\left(\frac{H_{1}}{\hbar}\right) \tau} e^{i\left(\frac{H_{2}}{\hbar}\right) \tau} e^{-i\left(\frac{H_{1}}{\hbar}\right) \tau} e^{-i\left(\frac{H_{2}}{\hbar}\right) \tau}\right| n\right\rangle\right)\right] \\
& =\frac{1}{2}\left[1-\operatorname{Re}\left(e^{i \frac{E_{n}^{1}}{\hbar} \tau}\left\langle\varphi_{n}(t=0) \mid \varphi_{n}(t=\tau)\right\rangle\right)\right]
\end{aligned}
$$

where $\left\{\varphi_{n}\right\}$ is a new basis defined by $\left|\varphi_{n}(t=0)\right\rangle \equiv \exp \left(-i \frac{H_{2}}{\hbar} \tau\right)|n\rangle$, and $\left|\varphi_{n}(t=\tau)\right\rangle \equiv \exp \left(-i \frac{H_{1}}{\hbar} \tau\right)\left|\varphi_{n}(t=0)\right\rangle . P_{2}$ no longer depends on $\omega_{M W}$ and $E_{H F}$ and therefore not on any $\Delta_{n}$ but only on the dynamics 2 . Ensemble averaging over a thermal ensemble therefore does not remove the fingerprints of the dynamics.

We performed echo spectroscopy on $\sim 10^{5}{ }^{85} R b$ atoms laser cooled to $\sim 20$

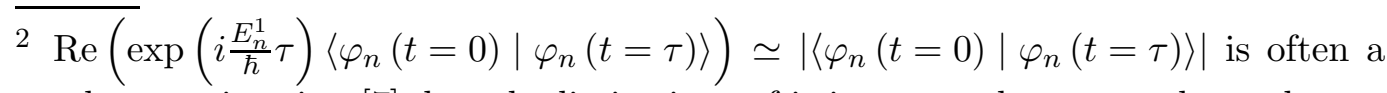
good approximation [7], but the limitations of it is not yet known to the authors. 


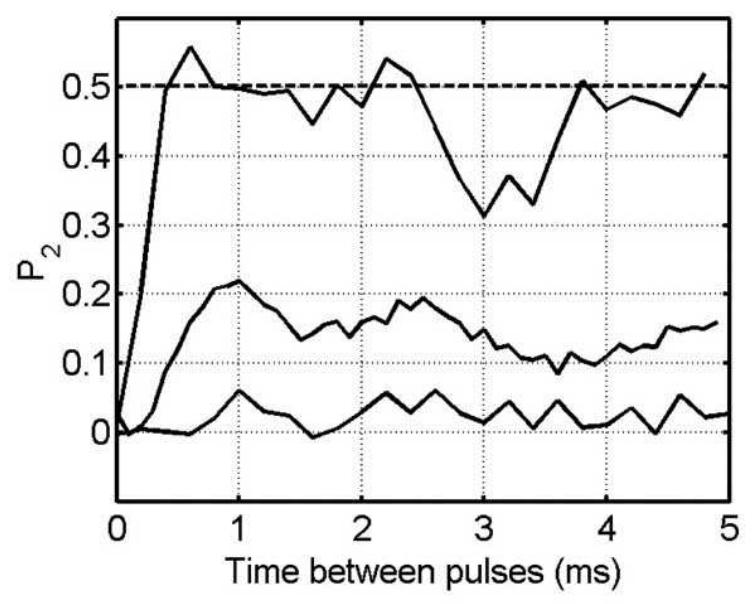

Fig. 2. Echo signal $P_{2}$ measured for three different trap laser wavelengths $\lambda$ close to the $D_{1}$ transition wavelength of $795 \mathrm{~nm}$. Lower curve: A "small perturbation" $(\lambda=805 \mathrm{~nm})$. A good echo signal $\left(P_{2}<<1 / 2\right)$ is seen almost independent of time between pulses. Middle curve: A "medium perturbation" $(\lambda=798.25 \mathrm{~nm})$. The echo signal oscillates around a value smaller than $1 / 2$ with partial revivals at $\tau=1 / 2 \tau_{\text {osc }}$ and $\tau=\tau_{o s c}$, where $\tau_{o s c}=3.6 \mathrm{~ms}$ is the measured trap oscillation frequency in the transverse direction. Upper curve: A "strong perturbation" $(\lambda=796.25 \mathrm{~nm})$. After a short time the echo signal completely disappears $\left(P_{2}=1 / 2\right)$, but partly revives again at $\tau=3.3 \mathrm{~ms}$, close to $\tau_{o s c}$.

$\mu K$ and then trapped in a $\sim 30 \mu K$ deep Gaussian optical trap with waist size of $\sim 50 \mu \mathrm{m}$. The procedure is detailed in [7]. The echo signal $P_{2}$ was measured as a function of time between pulses for different wavelengths of the trap laser, thereby changing the perturbation strength $\delta V$, while keeping the trap depth constant by adjusting the power of the trap beam. For a small perturbation a good echo $\left(P_{2}<<1 / 2\right)$ is seen independent of the time between pulses (see Fig. 2). For larger perturbation damped oscillations to a level smaller than $1 / 2$ are seen. An even larger perturbation causes a complete decay of the echo coherence $\left(P_{2}=1 / 2\right)$ followed by partial revivals, which are clear signatures of the dynamics. Two revivals (of different amplitude) are seen when the time between MW pulses is close to $\frac{1}{2} T_{\text {osc }}$ and $T_{\text {osc }}$, respectively, where $T_{\text {osc }}=3.5$ $\mathrm{ms}$ is the measured radial oscillation frequency of the trap (the longitudinal oscillation frequency is $\sim 1 s$, hence the longitudinal motion is essentially fixed for the duration of our experiment). The revival at $\frac{1}{2} T_{\text {osc }}$ corresponds to wavepacket oscillations excited by the symmetric change of the trap strength, and the stronger revival at $T_{o s c}$ corresponds also to the vertical sloshing of wavepackets induced by the vertical shift of the gravito-optical potential for $|2\rangle$ compared to $|1\rangle$ (in our experiments this vertical shift is of the order of a few $n m$, as compared to a trap cloud size of $\sim 50 \mu \mathrm{m}$ !). The lack of complete revival at $T_{o s c}$ is mainly due to anharmonicity of the Gaussian trap, but also small amount of decoherence due to spontaneous scattering of photons and noise may contribute. 


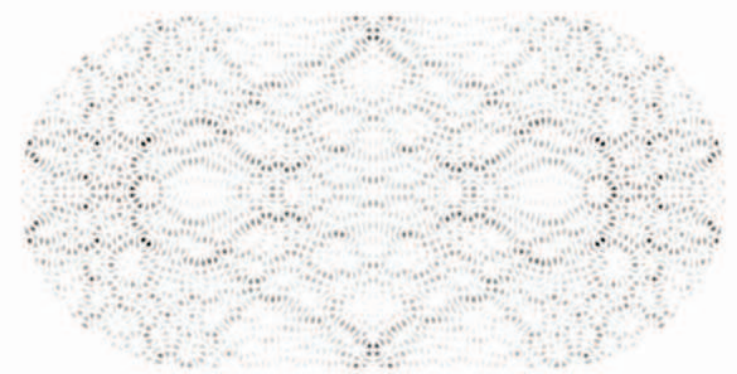

Fig. 3. Probability density plot of an eigenstate of a Bunimovich stadium $\left(k_{0} \simeq 100\right)$.

The sensitivity of our technique to map the quantum dynamics of a system is revealed here, since we see the dynamics due to a perturbation (kick), that is about three orders of magnitude smaller than $k_{B} T$.

\section{Eigenstate Stability and Atom Optic Billiards}

In Fig. 2 it is seen that for sufficient large detuning the long time asymptotic behavior of the echo signal from thermal atoms is smaller than $1 / 2$, and eventually becoming $\simeq 0$. This can be understood from Eq. 3: If $\delta V$ is small there will be, for each initial motional eigenstate $|n\rangle$ of $H_{1}$ a large matrix element for going into the equivalent motional eigenstate of $H_{2}$, since $H_{1}$ and $H_{2}$ are almost identical. If the time between pulses is large enough so the dynamics has dephased completely between them, then the contributions from other states will average to zero. Hence, the long time ensemble average of $P_{2}$ is simply given by $\overline{P_{2}}=\frac{1}{2}\left[1-\overline{\left|\left\langle n^{\prime}=n \mid n\right\rangle\right|^{4}}\right]$, where $|n\rangle$ and $\left|n^{\prime}=n\right\rangle$ are corresponding eigenstates of $H_{1}$ and $H_{2}$ respectively. In [7] a paraxial numerical calculation of $\overline{P_{2}}$, averaged over the $\sim 4 \times 10^{6}$ thermally-populated states of the trap yielded good quantitative agreement with measured long time echo signal.

Because of the importance of the eigenstate stability to the revival of coherence (good echo signal) and dynamics in the trap, we now calculate $\left|\left\langle n^{\prime}=n \mid n\right\rangle\right|$ for perturbations, that we assume approximate our inherent perturbation, i.e. the difference in dipole potential for the two hyperfine states, for different traps. Note, that since a large $\left|\left\langle n^{\prime}=n \mid n\right\rangle\right|$ also implies a large survival probability as defined in $[11,13,14]$, it also plays an important role in quantum dynamical studies.

Atom optic billiards are good candidates for trapping atoms for high precision spectroscopy, since the interaction with the trap light is small because atoms are confined mainly in the dark [12]. They have been used to study dynamics in the past, and have experimentally been demonstrated to display the features of ideal billiards, $[15,16,17,18]$. For our calculations we therefore approximate 


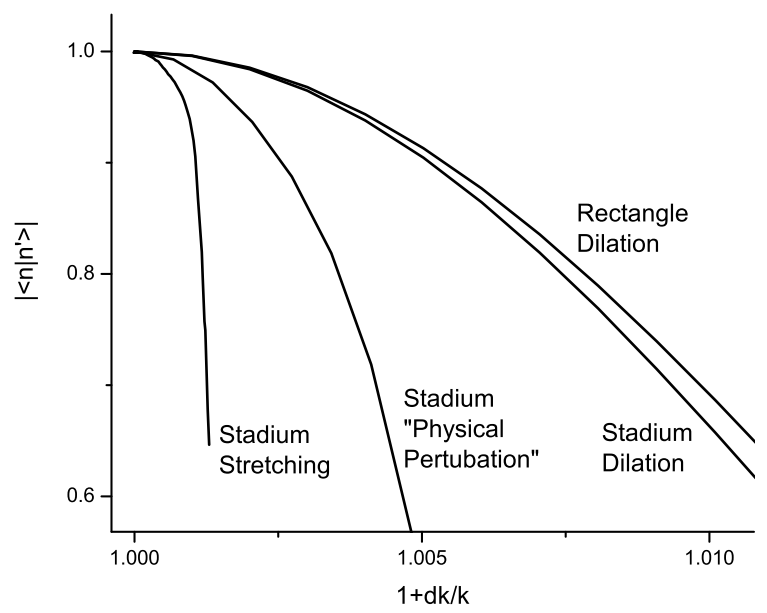

Fig. 4. $\left|\left\langle n^{\prime}=n \mid n\right\rangle\right|$ as a function of perturbation strength $1+\delta k / k$ for eigenstates with $k_{0} \simeq 100$. Three different perturbations are shown for the stadium eigenstate of Fig. 3, namely dilation, stretching and the "physical perturbation" (a dilation followed by a negative stretch). The rectangle state has $k_{x} \simeq k_{y}$. For dilation substantial reduction of $\left|\left\langle n^{\prime}=n \mid n\right\rangle\right|$ indeed occurs for $\delta k \simeq 1$, as expected from the intuitive discussion in the text.

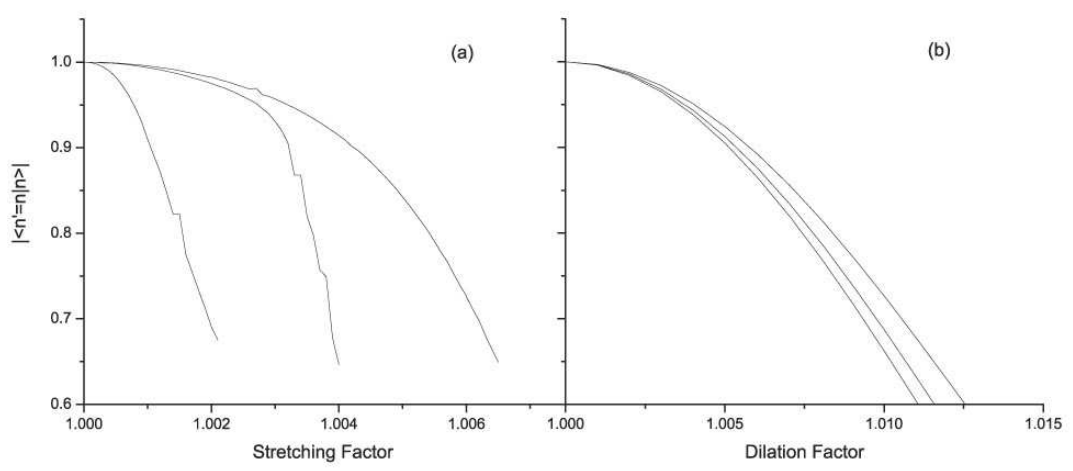

Fig. 5. $\left|\left\langle n^{\prime}=n \mid n\right\rangle\right|$ for three states of the stadium with $k_{0} \simeq 100$ as a function of perturbation strength. (a) Stretching: A strong dependence on the initial state is seen. (b) Dilation, for the same three stases as in (a): Only a weak dependence on the initial state with similar $k$ is seen.

the atom optics billiard with an ideal hard-wall billiard, and use the powerful technique of Vergini and Saraceno to numerically calculate highly excited eigenstates and eigenenergies [19]. For an unperturbed Bunimovich stadium [20] (two semicircles of radius $r=1$ connected by two straight lines of length $l=2$, for units defined such that $\hbar=2 m=1$ ) a plot of the probability density of a highly excited state is shown in Fig. 3. The size of its wave vector is $k_{0} \simeq 100\left(\nabla^{2} \psi=-k_{0}^{2} \psi\right)$ hence the wavefunction have on the order of few thousand antinodes.

Systems respond very different to different types of perturbations $[21,22,23,13,14]$. Perturbations of $V_{1}(\mathbf{x})$ that can be described as a change of coordinate system 


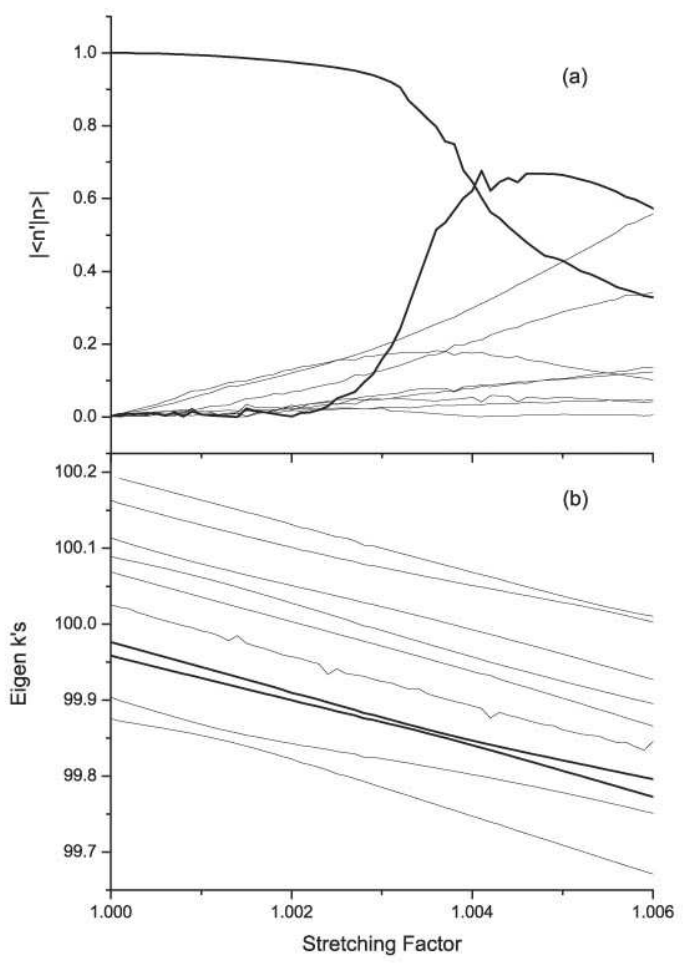

Fig. 6. (a) Matrix elements $\left(\left|\left\langle n^{\prime} \mid n\right\rangle\right|\right)$ between an initial state and its ten closest neighboring states with the same parity, as a function of stretching factor. (b) Eigen $k$ 's as a function of stretching factor, for the same states as (a). It is seen that when the state undergo avoided crossing it mixes strongly with the crossed state. Bold lines in (a) and (b) corresponds to the same states.

such as rotation, translation (of importance to the inverted pyramid discussed above) and dilation will give exceptional high $\left|\left\langle n^{\prime}=n \mid n\right\rangle\right|$. Intuitively this is clear since an eigenstate of $H_{1}$ under these three perturbations is also an eigenstate of $\mathrm{H}_{2}$ rotated, translated or dilated respectively. Therefore, to cause a significant drop in $\left|\left\langle n^{\prime}=n \mid n\right\rangle\right|$ the perturbation has to move the billiard wall distances on the order of typical distances between nodes in the eigenstate wave function, corresponding to $\delta k \simeq 1$ in case of dilation (see Fig. 4). On the other hand perturbations that changes the symmetry of the billiard can cause much faster changes in $\left|\left\langle n^{\prime}=n \mid n\right\rangle\right|$. This is illustrated in Fig. 4 where it is seen that dilating a Bunimovich stadium causes $\left|\left\langle n^{\prime}=n \mid n\right\rangle\right|$ to drop much slower than changing the lengths of the straight sections (this perturbation we call "stretching"). We are interested in the perturbation induced in an atom-optics billiard by changing the strength of the optical potential. This is best approximated by moving the billiard's wall a fixed distance perpendicular to itself. This perturbation, that we name "the physical perturbation", can be viewed as a dilation followed by a negative stretch, and it shows features between those of dilation and stretch (Fig. 4). 


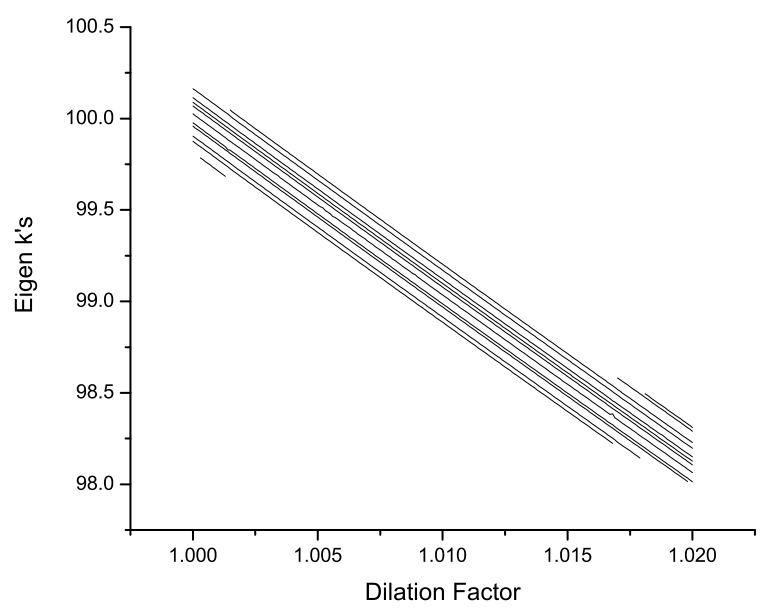

Fig. 7. $k$ as a function of dilation factor for eigenstates with $k_{0} \simeq 100$ in a stadium. Straight lines are observed as excepted.

In Fig $4\left|\left\langle n^{\prime}=n \mid n\right\rangle\right|$ for a rectangle is also shown in order to illustrate that the criteria stating that the dilation should move the wall of the billiard a distance of the order of the distance between nodes is valid not only for chaotic shapes but also for regular ones. For dilation in 1D systems this criteria is equivalent to the necessary criteria for substantial mixing from perturbation theory, namely that the perturbation energy should be comparable to the level separation. That this criteria also holds for dilating an integrable shape in 2D (where it corresponds to a perturbation energy of $\sim 100$ times the level spacing in our case) is also obvious since it is equivalent to two $1 \mathrm{D}$ systems. But its fulfilment for a chaotic system where variables cannot be separated is less obvious unless the above arguments are considered.

Figure 5 shows $\left|\left\langle n^{\prime}=n \mid n\right\rangle\right|$ as a function of perturbation strength for different eigenstates with $k_{0} \simeq 100$. It is seen that $\left|\left\langle n^{\prime}=n \mid n\right\rangle\right|$ is highly state dependent in case of stretching, but is not in case of dilation. Following the explanation given above, it is clear that states with almost the same $k_{0}$ have almost the same $\left|\left\langle n^{\prime}=n \mid n\right\rangle\right|$ for a given perturbation strength in case of dilation. The sudden drop in $\left|\left\langle n^{\prime}=n \mid n\right\rangle\right|$ in case of stretching is often associated with avoided crossings of energy levels, after which the two states involved still hold the major part of the probability. This is clearly seen from Fig. 6, where matrix elements to the ten closest states of same parity are shown for stretching'. In Fig. $6 \mathrm{~b} k$ as a function of stretching factor is shown for the same ten states. As seen, the avoided crossing of the two neighboring states (drawn in bold lines) occurs for the same stretch factor as the one causing strong mixing between them (Fig. 6)). In Fig. $7 k$ as a function of the dilation factor is presented, showing straight lines as expected. Matrix elements to close by states are 0 for dilation, in strong contrast to stretching shown in Fig. 6a [21].

3 Stretching is an even parity perturbation and selection rules therefore insures coupling only to states with the same parity 


\section{Conclusions}

We have analyzed echo spectroscopy of trapped ultra-cold atoms. Using this technique, we can combine the high precision of MW-spectroscopy with the high degree of control over experimental parameters provided by laser cooling and trapping techniques, to perform quantum dynamical studies even when many states are thermally populated. Based on this analysis we proposed a gravito-optical trap in which atoms in superposition states does not suffer from dephasing due to inhomogeneous Stark shifts.

We analyzed numerically the stability of eigenstates for several perturbations, and verified that the rapid drop in $\left|\left\langle n^{\prime}=n \mid n\right\rangle\right|$ seen for symmetry breaking perturbations is linked to avoided crossings of energy levels. The symmetry of the effective perturbations, caused by MW transitions in our experimental atom optics billiards was shown to depend on their shape. For example, rectangle or a circular billiard the physical perturbation corresponds approximately to pure dilation, thus enhancing the applicability of echo spectroscopy for these shapes, as compared to the Bunimovich stadium billiard discussed above.

Finally, the suppression of dephasing achieved with echo spectroscopy yields a dramatic increase in the coherence time for trapped atoms, that may find important applications for precision spectroscopy and quantum information processing.

\section{Acknowledgements}

This work was in part supported by Minerva Foundation, the United StatesIsrael Binational Science Foundation, and Foundation Antorchas. M. F. A. Acknowledges help from Dansk-Israelsk Studiefond til Minde om Josef og Regine Nachemsohn.

\section{References}

[1] J. P. Paz, W. H. Zurek, Environment-induced decoherence and the transition from quantum to classical, quant-ph/0010011 (October 1999).

[2] M. Nielsen, I. L. Chuang, Quantum Information and Quantum Computation, Cambridge University Press, 2001.

[3] E. L. Hahn, Spin echoes, Phys. Rev. 80 (4) (1950) 580-601.

[4] N. A. Kurnit, I. D. Abella, S. R. Hartmann, Observation of a photon echo, Phys. Rev. Lett. 13 (19) (1964) 567-568. 
[5] L. Allen, J. H. Eberly, Optical Resonance and Two-Level Atoms, Dover Publications, Inc., New York, 1987.

[6] F. B. J. Buchkremer, R. Bumke, H. Levsen, G. B. W., Ertmer, Wave packet echoes in the motion of trapped atoms, Phys. Rev. Lett. 85 (15) (2000) 31213124 .

[7] M. F. Andersen, A. Kaplan, N. Davidson, Echo spectroscopy and quantum stability of trapped atoms, Phys. Rev. Lett. In Press.

[8] A. Kaplan, M. Andersen, N. Davidson, Suppression of inhomogeneous broadening in rf spectroscopy of optically trapped atoms, Phys. Rev. A 66 (2002) 045401-045404.

[9] N. F. Ramsey, Molecular Beams, Clarendon Press, 1956.

[10] C. Cohen-Tannoudji, J. Dupont-Roc, G. Grynberg, Atom-photon interactions, John Wiley \& Sons, New York, 1992.

[11] A. Peres, Stability of quantum motion in chaotic and regular systems, Phys. Rev. A 30 (4) (1984) 1610-1615.

[12] N. Friedman, A. Kaplan, N. Davidson, Dark optical traps for cold atoms, Adv. At. Mol. Opt. Phys. 48 (2002) 99-151.

[13] D. A. Wisniacki, E. Vergini, H. M. Pastawski, F. M. Cucchietti, Sensitivity to perturbations in a quantum chaotic billiard, arXiv:nlin.CD/0111051 (2001).

[14] D. Wisniacki, Short time decay of the loschmidt echo, nlin.CD/0208044 (August 2002).

[15] V. Milner, J. L. Hanssen, W. C. Campbell, M. G. Raizen, Optical billiards for atoms, Phys. Rev. Lett. 86 (8) (2001) 1514-1517.

[16] N. Friedman, A. Kaplan, D. Carasso, N. Davidson, Observation of chaotic and regular dynamics in atom-optics billiards, Phys. Rev. Lett. 86 (8) (2001) 15181521.

[17] A. Kaplan, N. Friedman, M. Andersen, N. Davidson, Observation of islands of stability in soft wall atom-optics billiards, Phys. Rev. Lett. 87 (27) (2001) 274101[1]-274101[4].

[18] M. F. Andersen, A. Kaplan, N. Friedman, N. Davidson, Stable islands in chaotic atom-optics billiards, caused by curved trajectories, J. Phys. B: At. Mol. Opt. Phys. 35 (9) (2002) 2183-2190.

[19] E. Vergini, M. Saraceno, Calculation by scaling of highly excited states of billiards, Phys. Rev. E 52 (3) (1995) 2204-2207.

[20] L. A. Bunimovich, On the ergodic properties of nowhere dispersing billiards, Commun. Math. Phys. 65 (1979) 295-312.

[21] A. Barnett, D. Cohen, E. J. Heller, Deformations and dilations of chaotic billiards: Dissipation rate, and quasiorthogonality of the boundary wave functions, Phys. Rev. Lett. 85 (7) (2000) 1412-1415. 
[22] D. Cohen, E. J. Heller, Unification of perturbation theory, random matrix theory, and semiclassical considerations in the study of parametrically dependent eigenstates, Phys. Rev. Lett. 84 (13) (2000) 2841-2844.

[23] D. Cohen, A. Barnett, E. J. Heller, Parametric evolution for a deformed cavity, Phys. Rev. E 63 (2001) 046207[1]-046207[12]. 